\title{
Endovascular retrieval of ruptured and dislocated vascular catheter
}

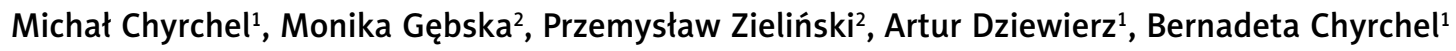 \\ ${ }^{1} 2^{\text {nd }}$ Department of Cardiology, Jagiellonian University Medical College, Krakow, Poland \\ ${ }^{2}$ Department of Cardiology, District Hospital, Radomsko, Poland
}

Adv Interv Cardiol 2018; 14, 2 (52): 216-217

DOI: https://doi.org/10.5114/aic.2018.76422

A 61-year-old female patient was admitted to the cardiology department due to dyspnea and atypical chest pain. She had cervical cancer with metastasis to the lungs which was diagnosed 2 years earlier. The patient was undergoing palliative chemotherapy (vinorelbine) administered through a silicone catheter (Celsite - implantable access port system; B Braun Medical, France) localized in the right subclavian vein. On admission, the patient was hemodynamically stable (blood pressure: 120/80 mm Hg, heart rate: 80 bpm, saturation $98 \%$ ). Laboratory tests revealed anemia (Hb: $10.1 \mathrm{~g} / \mathrm{dl}, \mathrm{Ht}: 31.1 \%$ ) and elevated serum creatinine $\left(1.2 \mathrm{mg} / \mathrm{dl}\right.$, eGFR $\left.47.0 \mathrm{ml} / \mathrm{min} / 1.73 \mathrm{~m}^{2}\right)$. Pulmonary embolism was excluded using echocardiography and D-Dimer measurement. In echocardiography a longitudinal shadow was found in the heart protruding to the inferior vena cava. Therefore an abdominal X-ray was performed (Figure $1 \mathrm{~A}$ ). Examination confirmed the presence of a ruptured chemotherapy catheter. A $17.5 \mathrm{~cm}$ long catheter was located in the inferior vena cava and right atrium. Due to the risk of thrombus-embolic complications the patient was qualified for endovascular removal of the catheter.

After local anesthesia the right femoral vein was punctured. A $7 \mathrm{Fr}$ vascular sheath was introduced and a 7 Fr Judkins right guiding catheter (Medtronic, USA) was located below the lower tip of the catheter. An Andrasnare AS-10 device with atraumatic $10 \mathrm{~mm}$ nitinol loop (Andramed $\mathrm{GmbH}$, Germany) was introduced through the guiding catheter. The device loop was positioned at the very end of the catheter, which enabled coaxially pulling out the ruptured catheter through the $7 \mathrm{Fr}$ sheath (Figures $1 \mathrm{~B}-\mathrm{D})$. Fluoroscopy time was $9 \mathrm{~min} 12 \mathrm{~s}$ with a radiation dose of $0.2 \mathrm{~Gy}$. A total of $20 \mathrm{ml}$ of contrast media was used. There were no periprocedural complications. The next day, the remaining part of the broken vascular port chamber was removed surgically from subcutane- ous tissue in the right subclavian vein region. The patient was discharged home on the fifth day.

Subcutaneous venous catheters are commonly used in patients with cancer. Located in big veins, they are used for fluid infusion, parenteral nutrition, and administration of chemotherapy agents [1-3]. Early complications associated with catheters occur immediately after the implantation. They can be related to clinical conditions of the patient or to surgical factors. These include skin or vascular perforation, hemothorax, incorrect positioning of the catheter, and improper anchoring of the catheter with early dislocation. The rate of early complications is below $4.5 \%$ [4]. The late complications include infection of the subcutaneous pocket or the port system, skin infection, sepsis, drug extravasation, mechanical malfunction, skin necrosis, catheter thrombosis, catheter disconnection and embolization, and venous thrombosis. The rate of migration of the ruptured catheter ranges from $0.2 \%$ to $8 \%$ [4]. The mechanism and the exact time of catheter rupture in the present case is unknown. Dislocation of the separated catheter fragment to the cardiovascular system is not frequent. It is associated with possible severe clinical consequences (life-threatening arrhythmias, sepsis, thrombus formation, endocarditis, heart perforation or even death) [2, 4]. Therefore the dislocated catheter should be urgently removed from vascular circulation. The recommended approach for foreign body removal is minimally invasive endovascular treatment under fluoroscopy with snaring devices [3, 5]. Minimally invasive techniques present outstanding results and may minimize morbidity and mortality [3]. The remaining catheter part in the subcutaneous tissue can be easily removed surgically under local anesthesia.

\section{Conflict of interest}

The authors declare no conflict of interest.

\section{Corresponding author:}

Michat Chyrchel MD, PhD, $2^{\text {nd }}$ Department of Cardiology, Jagiellonian University Medical College, 17 Kopernika St, 31-501 Krakow, Poland, phone: +48 1242471 81, e-mail: mchyrchel@gmail.com

Received: 9.03.2018, accepted: 11.04.2018. 


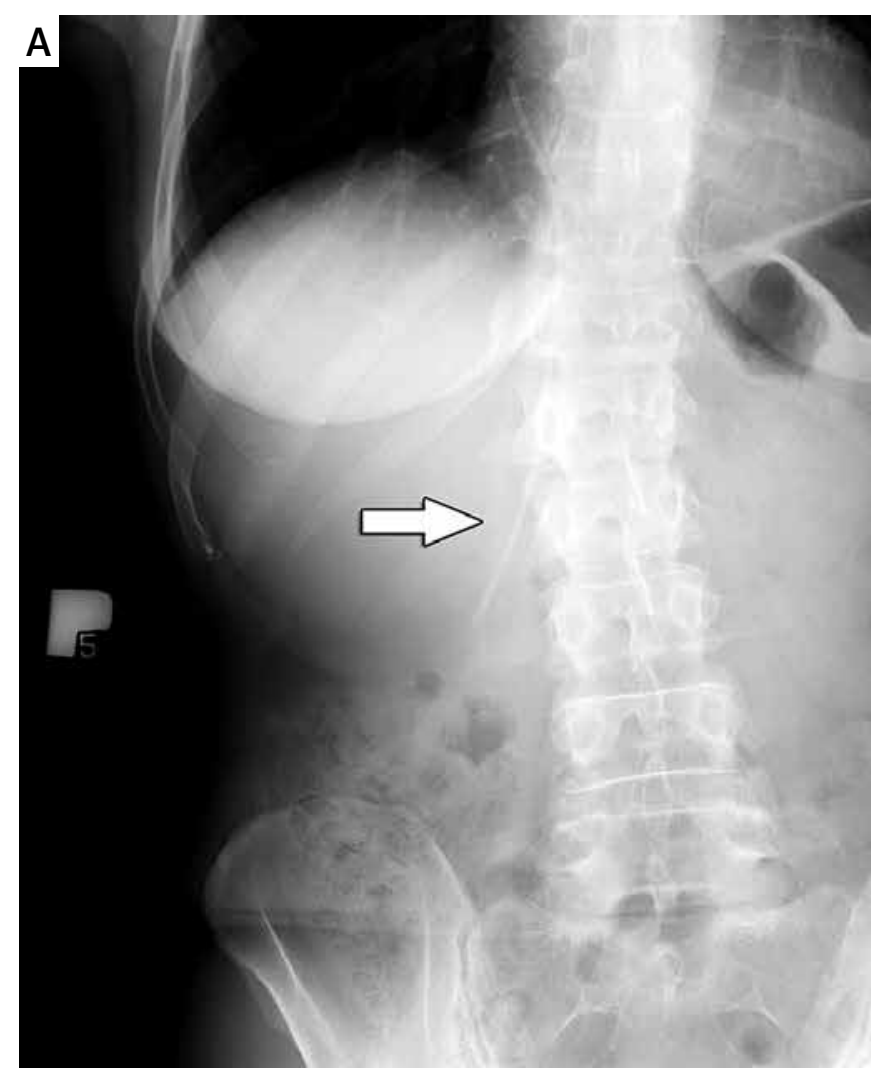

B

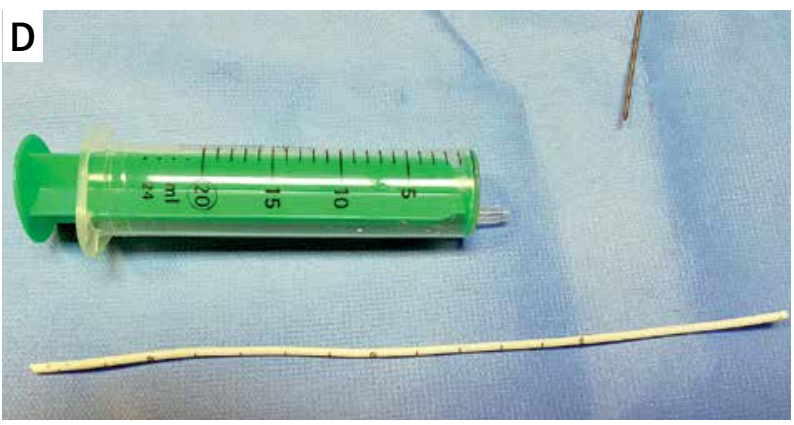

Figure 1. A - An abdominal X-ray shows dislocated part of the chemotherapy catheter in the inferior vena cava and right atrium (arrow). B - Snare loop captures the catheter (arrow). C - Device loop at the end of the catheter (arrow). D - Removed catheter

\section{References}

1. Cheng CC, Tsai TN, Yang CC, et al. Percutaneous retrieval of dislodged totally implantable central venous access system in 92 cases. Experience in a single hospital. Eur J Radiol 2009; 69: 346-50.

2. Kalińczuk $九$, Chmielak Z, Dębski A, et al. Percutaneous retrieval of centrally embolized fragments of central venous access devices or knotted Swan-Ganz catheters. Clinical report of 14 retrievals with detailed angiographic analysis and review of procedural aspects. Adv Interv Cardiol 2016; 12: 140-55.

3. Filippou DK, Tsikkinis C, Filippou GK, et al. Rupture of totally implantable central venous access devices (Intraports) in patients with cancer: report of four cases. World I Surg Oncol 2004; 2: 36.

4. Intagliata E, Basile F, Vecchio R. Totally implantable catheter migration and its percutaneous retrieval: case report and review of the literature. G Chir 2016; 37: 211-5.

5. Yen HJ, Hwang B, Lee PC, et al. Retrieval of different types of central venous catheter fragment: experience in 13 cases. Angiology 2006; 57: 347-53. 